\title{
A case report of human infestation with pubic louse (Pthirus pubis) in Sarab city, Iran
}

\author{
Garedaghi Yagoob ${ }^{1 *}$
}

\begin{abstract}
Lice were collected from 47 years old male worker on his complaint for itching and red rashes on body. The lice thus collected were subjected to microscopic examinations and identified as Phthirus pubis, commonly known as the crab louse, an ecto-parasite which is only found on humans and requires human blood to survive. The infested parts of the body of worker was treated with $1 \%$ Permethrin cream, that was applied for 10 minutes and then rinse off with water. Second treatment was done on day and after 14 days no viable lice and nits were seen on the body of the victim
\end{abstract}

Key words: Human Louse, Phthirus pubis, Sarab city, Iran.

\section{Introduction}

The sucking lice (order Anoplura) are small, wingless and dorso-ventrally flattened insects. This order contains several families of which Pediculidae and Pthiridae parasitize human, so called human lice. There are two species of Pediculidae, head and body lice (Pediculus capitis and Pediculus humanus) and a species of Pthiridae, pubic louse (Pthirus pubis) that infect humans (1).

Lice infestations have been prevalent among humans for many centuries and it has been revealed that lice infestations are associated with lack of hygiene and poverty in human societies.Phthirus pubis, commonly known as the crab louse, is an ectoparasite which is only found on humans and requires human blood to survive.

The crab louse does not fly and jump, only crawl slowly, so it usually needs close and prolonged contact to infest new hosts, most often through sexual contact, although it may occur via close nonsexual contact, including toilet seats, clothing, or bedding (1). Pediculosis pubis (PP) is infestation with pubic lice of the species Phthirus pubis.

There are no ratial differences in its incidence and the infestation is generally common. Direct contact is the primary source of transmission. In adults, PP most frequently occurs as a sexuallytransmitted disease (STD), commonly associated with other STDs. However, transmission may occur from sheets and clothing. Phthirus pubis habituates regions that are rich in apocrine glands, so predilection sites are pubic area, axillae and eyelashes.

Scalp hair, beard, moustache, and in hirsute individuals short hairs of the thighs, trunk and perianal area may be involved. Phthirus pubis in eyelashes and periphery of the scalp is mainly found in children, probably as the result of contact with an infected parent (2). Pubic lice feed and reproduce on the human host cementing their nits to the hair shaft $1 \mathrm{~cm}$ from the skin surface and nits hatch in 8 to 10 days. The majority of patients complain of pruritus. Pruritus is moderate.

Typical clinical findings are blue to grey macules (sky-blue spots), maculae ceruleae, sized from several millimeters to several centimeters (2).

Excoriations are not commonly found. Secondary infection due to excoriations can lead to local lymphadenitis and fever. Pthirus pubis is a specific parasite of humans, and although its transfer to a dog has been recorded (3) it cannot survive off the host for more than 24 hours. The pubic louse is spread primarily through close physical or sexual contact, with about $95 \%$ of sexual contacts becoming infested (4).

The diagnosis is confirmed by microscopic examination of the plucked hair to identify the nits with vital nymphs and hatched empty cases. Lice are difficult but possible to see with close inspection or magnification. Additionally, dermoscopy allows to differentiate nits with vital nymphs from empty cases and to identify pubic lice (5). Infestations with pubic lice are more common in people of low socioeconomic status (6) and are frequently associated with the presence of other sexually transmitted infections (7).

It has been stated than the population with the highest incidence of pubic lice is similar to that with a high incidence of gonorrhea and syphilis: single persons and those between 15 and 25 years of age (8). 


\section{Case}

A 47 year old male worker in sarab city of Iran, came to the Department of Parasitology, Islamic Azad University, Tabriz, Iran, suffering from constant itching on the both upper arms lasting for two weeks and claimed that due to close contact with a dog he was getting tick infestation. Physical examination of the patients revealed typical clinical findings-blue to grey macules on both arms with very small lice (Figure-1).

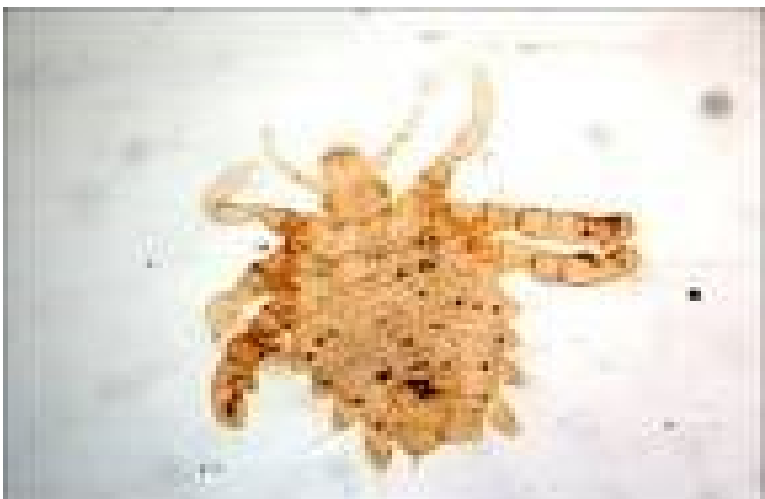

Figure 1. Photograph of Female pubic louse (Phthirus pubis).

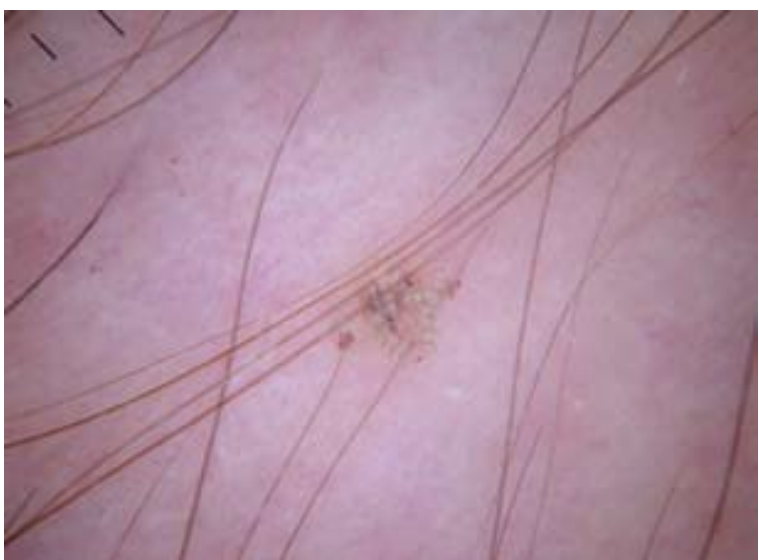

Figure 2. Photograph of pubic louse (Phthirus pubis) nit cemented with hair.

Further investigation revealed that there was severe irritation or itching on the pubic area. During investigation patient admitted that he had used his friend's bed and it could be the source of infestation. Symptomatically patient also indicated pruritus, itching, anorexia, lethargy, weakness and sexual depression.

The lice and nits were removed manually with a pair of tweezers and were placed on a glass slide; a drop of glycerin was used as a mounting media and covered with a glass cover slip. Results of microscopic examination revealed the presence of Phthirus pubis (Pubic lice) and their nits cementing with the hairs (Figure-2). Male and female Phthirus pubis was identified on their morphological Characters (Fig 1 and 2). The infested parts of the body of worker was treated with $1 \%$ Permethrin cream, that was applied for 10 minutes and then rinse off with water. Second treatment was done on day 10 and after 14 days no viable lice and eggs were seen on the body of the victim

\section{Discussion}

Normally crab louse infestation is limited to pubic area, but other hair- bearing sites such as thighs, axillae, mustache, beard, eyelashes, eyebrows and trunk can be involved $(9,10)$. The first case was reported in 1892 and most reported cases were found in children. The youngest reported patient was a 6week- old male infant $(11,12)$.

Goldman found a female to male ratio of $2: 1$ $(13,14,15)$. On the bases of present case, it may be concluded that 02 application of $1 \%$ Permethrin cream, for 10 minutes on day 1 and 10 gives significant result, as no viable lice and eggs were seen on the body of the victim.

\section{Acknowledgments}

The author would like to thanks Tabriz Branch, Islamic Azad University for the laboratory Instruments supports of this research, and all persons that aid me in this project.

\section{References}

1. Goddard J,. Lice. In: Physician's guide to arthropods of medical importance. 3rd ed. Florida: CRC Press LLC. 2000; 213-216

2. Stone SS, Scabies and pediculosis. Fitzpatrick's Dermatology in General Medicine. 6th Ed. NewYork: McGraw Hill. 2003; 2283-9. Frye $\mathrm{F} L$ and Furman DP,. Phthiriasis in a dog. J Am Vet Med Assoc. 1965; 152:1113.

Letau L A,. Nosocomial transmission and infection control aspects of parasitic and ectoparasitic diseases. Part III. Ectoparasites/ summary and conclusions. Infect Control Hosp Epidemiol. 1991; 12:179 -185.

5. Chuh A, Lee A, Wong W, Ooi C and Zawar V,. Diagnosis of pediculosis pubis: a novel application of digital epiluminescence dermatoscopy. J Eur Acad Dermatol Venereol. 2007; 21:837-8.

6. Gillis D, Slepon R, Karsenty E and Green MS, Sociodemographic factors associated with pediculosis capitis and pubis among young adults in the Israel Defense Forces. Public Health Rev. 1990;18:345-350.

7. Garedaghi Yagoob, Flea Infestation in Farm Animals and its Zoonotic Importance in East-Azerbaijan Province, American Journal of Animal and Veterinary Sciences 6 (4): 193-196, 2011.

8. Billstein SA, Pubic lice. In: Holmes KK, Sparling PF, Mardh P-A, et al, eds. Sexually Transmitted Diseases. 3rd ed. New York: McGraw- Hill.1999. 641-644. 
9. Brown TJ, Yen-Moore A and Tyring S.K,. An overview of sexually transmitted diseases. Part II. J Am Acad Dermatol. 1999; 41: 61-677.

10. Javaid A. Gadahi, Abdullah G. Arijo, Bachal Bhutto Nasreen Akhter. Human Louce Infestation: A Case Report. IJAVMS, Vol. 4, Issue 3, 2010: 63-66.

11. Singh S, Singh N and Ray JC,. Phthirus pubis infestation of the scalp: report of three cases. Rev nfect Dis. 1990; 12: 560 .

12. Routh HB, Mirensky YM, Parish LC and Witkowski J A, Ectoparasites as sexually transmitted diseases. Semin Dermatol. 1994;13:243-247.
13.

Silburt BS and Parsons WL,. Scalp infestation by Phthirus pubis in a 6-week-old infant. Pediatr Dermatol. 1990; 7: 205-207.

14.

Poudel, S.K. and Barker, S.C. (2004). Infestation of people with lice in Kathmandu and Pokhara, Nepal. Medical and Veterinary Entomology 18: 212-213.

15. Service, M.W. (1996). Medical Entomology. St. Louis: Mosby, 189-93.

Copyright (C) 2014 The Author(s); This is an open-access article distributed under the terms of the Creative Commons Attribution License (http://creativecommons.org/licenses/by/4.0), which permits unrestricted use, distribution, and reproduction in any medium, provided the original work is properly cited. 